\title{
Headache characteristics in COVID-19 pandemic-a survey study
}

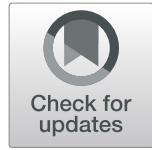

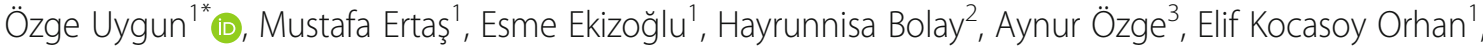 \\ Arif Atahan Çağatay ${ }^{4}$ and Betül Baykan ${ }^{1^{*}}$
}

\begin{abstract}
Background: Headache is the most common COVID-19-related neurological symptom. We aimed to reveal diagnostic clues of headache for COVID-19 infection and to investigate the course of primary headaches during the pandemic.

Methods: We developed a detailed web-based questionnaire screening the characteristics and course of headaches besides clinical COVID-19 features. The participants were grouped according to being diagnosed with COVID-19 infection or not, and having previous or new-onset headaches. The COVID-19 related headache features and their associations with other clinical features were investigated. A binary logistic regression model was performed to differentiate the characteristics of headache related to COVID-19.
\end{abstract}

Findings: A total of 3458 participants (2341 females;67.7\%, 1495 healthcare workers;43.2\%) with a mean age of $43.21 \pm$ 11.2 years contributed to the survey. Among them, 262 participants had COVID-19 diagnosis and 126 (48.1\%) were male. The rate of males in the group without COVID-19 was 31\% (991 out of 3196 participants) showing significant gender difference between groups $(p<0.000)$. COVID-19 related headaches were more closely associated with anosmia/ageusia and gastrointestinal complaints $(p<0.000$ and $p<0.000)$, and showed different characteristics like pulsating, pressing, and even stabbing quality. Logistic regression analyses showed that bilateral headache, duration over $72 \mathrm{~h}$, analgesic resistance and having male gender were significant variables to differentiate COVID-19 positive patients from those without COVID-19 ( $p=0.04$ for long duration and $p<0.000$ for others). A worsening of previous primary headaches due to the pandemic-related problems was not reported in the majority of patients.

Interpretation: Bilateral, long-lasting headaches, resistance to analgesics and having male gender were more frequent in people with COVID-19 in conjunction with anosmia/ageusia and gastrointestinal complaints. These features may be helpful for diagnosing the headache related to COVID-19 during the pandemic.

Keywords: COVID-19 infection, Headache, Migraine

\section{Introduction}

Coronavirus disease-19 (COVID-19) caused by the severe acute respiratory syndrome coronavirus 2 (SARSCOV-2) first emerged in Wuhan towards the end of 2019 [1]. The pandemic is now influencing the whole

\footnotetext{
*Correspondence: ozz_uygun@hotmail.com; betul.baykan.baykal@gmail.com ${ }^{1}$ Department of Neurology, Istanbul Faculty of Medicine, Istanbul University, Çapa, Istanbul, Turkey

Full list of author information is available at the end of the article
}

world with a large number of deaths, besides many other medical and social consequences. The most common clinical picture of COVID-19 is characterized by manifestations of the respiratory system, as the name of the virus implies. However, many other complaints such as anosmia, ageusia, diarrhea and also headache have been noted in the clinical spectrum, with an increasing number of patients [2]. A handful of reports disclosed that

\section{$\triangle B M C$}

(c) The Author(s). 2020 Open Access This article is licensed under a Creative Commons Attribution 4.0 International License, which permits use, sharing, adaptation, distribution and reproduction in any medium or format, as long as you give appropriate credit to the original author(s) and the source, provide a link to the Creative Commons licence, and indicate if changes were made. The images or other third party material in this article are included in the article's Creative Commons licence, unless indicated otherwise in a credit line to the material. If material is not included in the article's Creative Commons licence and your intended use is not permitted by statutory regulation or exceeds the permitted use, you will need to obtain permission directly from the copyright holder. To view a copy of this licence, visit http://creativecommons.org/licenses/by/4.0/ The Creative Commons Public Domain Dedication waiver (http://creativecommons.org/publicdomain/zero/1.0/) applies to the data made available in this article, unless otherwise stated in a credit line to the data. 
headache is among the COVID-19-related symptoms, showing highly variable rates across the studies [3-6].

There are some studies and reviews highlighting that the most common neurological symptom is headache, often accompanied by high fever, moreover headache can occasionally be seen alone as the first sign of the disease [2, 7]. A recent meta-analysis reported that the prevalence of headache was $10.9 \%$ with a high level of heterogeneity [8]. Therefore, detailed questioning of the presence of headaches in patients who are admitted to the emergency and outpatient departments is still an important step in the prompt recognition of the infection in some patients.

In clinical observations and small case series, COVID19 related headache was described as acute at onset, usually occurring in a different character unlike previous headaches $[9,10]$. However, there are no systematic data on the headache characteristics.

Another important problem is the situation of patients with previous severe headaches like migraine in the COVID-19 pandemic era. COVID-19 itself or its psychosociological effects may cause more headache burden, along with the problems of quarantine and these points are not yet investigated thoroughly.

In this study, we aimed to reveal COVID-19 related headache characteristics and its associations. Our secondary aims were to investigate the features and course of pre-existing headaches as well as new-onset ones in participants without COVID-19 but having headaches during the pandemic.

\section{Methods}

\section{Design of a web-based questionnaire}

After the emergence of COVID-19 for the first time in March 2020 in our country, the complaints of peculiar headaches in consulted cases and some distinctive headache features in admitted patients attracted our attention. For this reason, a detailed questionnaire was developed focusing on headache characteristics, besides some clinical COVID-19 features by a panel of headache experts, after rounds of online discussions.

The number of the questions was tried to be kept in an acceptable limit to optimize the attention during answering the survey and the survey was tested before its submission by five patients for clarity of the questions. The web-based, user-friendly technical design was planned by the experts of the Istanbul University Department of Informatics.

The features of previous headaches were screened and headache diagnosis was made according to International Classification of Headache Disorders, 3rd version (ICHD-3 criteria) [11]. Possible changes in the headache course during the pandemic along with perceived precipitating factors and related characteristics were queried with scrutiny. Important characteristics such as the duration, frequency, course, localization, the severity of headaches, and treatment response were asked separately before and during the COVID-19 pandemic. The questionnaire has also asked if the participant had been diagnosed with COVID-19 as well as the symptoms of COVID-19 infection in detail for relevant patients. Even if the patient was not diagnosed with COVID-19, factors secondary to the pandemic lifestyle as possible triggers of headache, such as wearing a mask, having the fear of infection, etc. were evaluated. The questionnaire has included a total of 39 questions, and based on four different parts, in terms of demographics, previous headache features, reported COVID-19 infection features, and details of the headache course after the pandemic.

The participants were invited by means of social media (using Twitter, Instagram or Whatsapp) using a webbased link, suitable also for smart phones, after the Ethics Committee approval (17.04.2020/520). Discharged COVID-19 patients of the Istanbul University Faculty of Medicine Hospital were invited by text messages to volunteer the survey, in order to increase the number of patients with COVID-19 infection-related headache.

\section{Inclusion and exclusion criteria}

Eligible participants older than 18-years who had a COVID-19 infection experiencing headache among their symptoms or who had any type of headache before the pandemic, or developed new-onset headaches were included. The volunteers who participated in the survey were grouped according to being diagnosed with COVID-19 infection or not. In our country's protocol, only those patients who have polymerase chain reaction (PCR) positivity, are diagnosed with COVID-19. Especially, healthcare workers were encouraged to attend the study. Figure 1 shows the flowchart of our study. Surveys completed by participants under age of 18 or those reporting inconsistent responses were excluded. Participants without headache during the pandemic were not included in the statistical analysis.

\section{Study period}

The duration of survey implementation was planned as 15 days, starting on 1 May 2020. Although reaching a high number in a few hours, the survey was continued to the end of this period to include more patients with COVID-19 related headaches.

\section{Statistical analysis}

Descriptive analyses were applied for four main groups of participants (Fig. 1, Table 1); the participants with or without COVID-19 were compared in relation to the presence or absence of previous headaches before the pandemic of COVID-19 in regard to headache 


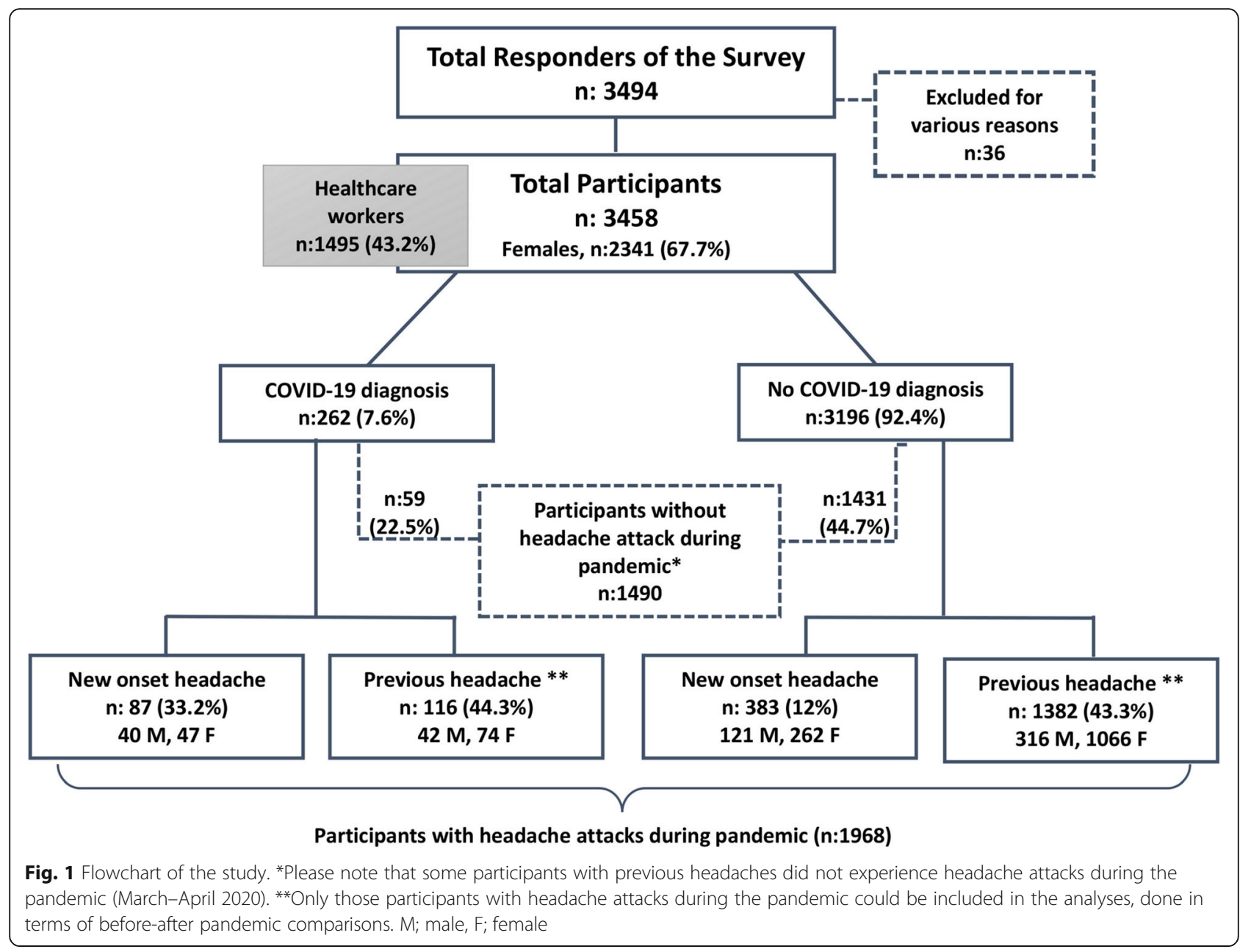

characteristics by the chi-square test and t-test where appropriate. A binary logistic regression model was performed to explore the differentiating headache variables between COVID-19 positive and negative cases. The odds ratios were calculated for significant infectionrelated features, such as anosmia/ageusia and gastrointestinal complaints like diarrhea. IBM SPSS Statistics Version 22 was used and $p<0.05$ was considered as statistically significant.

\section{Results}

A total of 3458 participants (2341 females) contributed to our survey. Of this main group with and without headache during the pandemic, 262 patients were diagnosed with COVID-19 by PCR (Fig. 1). These 262 patients were grouped as COVID-19 positive including 136 females (51.9\%) and 89 healthcare workers (9.8\%).

The characteristics of patients with COVID-19 infection In the COVID-19 positive group, the rate of males was $48.1 \%$ (126 out of 262 patients), whereas in the COVID19 negative group this rate was 31\% (991 out of 3196 participants), showing a significant gender difference $(p<0.000)$. Headaches lasting over $72 \mathrm{~h}$ were reported by $10.3 \%$ (27 out of 262 participants) of COVID-19 infected patients versus $4.1 \%$ (130 out of 3196 participants) of the COVID-19 negative group $(p<0.000)$.

In this study, 1968 participants with or without COVID-19 infection reported headache attacks during the pandemic. Among them 714 (36.3\%) had migraine and 1077 (54.7\%) participants were diagnosed with tension type headache (TTH) according to ICHD-3 criteria. The headache characteristics, accompanying features, treatment responses of these patients experiencing headache during the pandemic, according to having or not having prior headaches were shown in Table 1, comparatively. Osmophobia was more frequently reported in the group with COVID-19. Most of the COVID-19 cases (71\%) experienced nausea and gastrointestinal symptoms, for example, diarrhea/stomachache were present in more than half of them. Interestingly, pulsating quality was more pronounced in patients with previous headaches in the group with COVID-19 in comparison to the group with history of new onset headache. 
Table 1 Demographic features of participants and characteristics of headache experienced during the pandemic period

\begin{tabular}{|c|c|c|c|c|c|c|c|c|}
\hline \multirow[t]{2}{*}{$(n=1968)$} & \multicolumn{3}{|c|}{ COVID-19 positive $^{a}$} & \multicolumn{3}{|l|}{ No COVID-19 } & \multirow[b]{2}{*}{$\begin{array}{l}\text { p (1- } \\
3) \dagger\end{array}$} & \multirow[b]{2}{*}{$\begin{array}{l}p(2- \\
4) \neq\end{array}$} \\
\hline & $\begin{array}{l}\text { Prior headache } \\
(n=116)\end{array}$ & $\begin{array}{l}\text { No prior headache } \\
(n=87)\end{array}$ & $p$ & $\begin{array}{l}\text { Prior headache } \\
(n=138)\end{array}$ & $\begin{array}{l}\text { No prior headache } \\
(n=383)\end{array}$ & & & \\
\hline Age year,mean (SD) & $39.7(11)$ & $38.2(11)$ & .370 & $41.0(10)$ & $42.2(11)$ & .061 & .175 & .004 \\
\hline Sex (male \%) & 36.2 & 46.0 & .104 & 22.9 & 31.6 & .000 & .001 & .008 \\
\hline Healthcare worker (\%) & 44.0 & 43.7 & .541 & 46.1 & 48.3 & .239 & .366 & .255 \\
\hline $\begin{array}{l}\text { Headache duration hours mean } \\
\text { (SD) }\end{array}$ & $52.1(57)$ & $45.3(53)$ & .430 & $38.4(53)$ & $45.9(63)$ & .039 & .014 & .949 \\
\hline \multicolumn{9}{|l|}{ Headache characteristics } \\
\hline Pulsating \% & 50.9 & 32.5 & .008 & 55.5 & 42.5 & .000 & .202 & .064 \\
\hline Pressing \% & 31.9 & 43.7 & .058 & 29.0 & 38.6 & .000 & .289 & .227 \\
\hline Fiery $\%$ & 2.6 & 2.3 & .633 & 2.7 & 5.0 & .022 & .625 & .219 \\
\hline Stabbing \% & 12.9 & 16.1 & .330 & 11.1 & 11.2 & .497 & .315 & .142 \\
\hline \multicolumn{9}{|l|}{ Accompanying symptoms } \\
\hline Nausea \% & 70.8 & 71.3 & .538 & 53.5 & 52.9 & .450 & .000 & .002 \\
\hline Phonophobia \% & 67.0 & 67.9 & .509 & 71.2 & 66.6 & .056 & .198 & .465 \\
\hline Photophobia \% & 63.9 & 63.0 & .508 & 60.5 & 51.2 & .002 & .276 & .038 \\
\hline Osmophobia \% & 39.0 & 50.0 & .090 & 30.4 & 28.5 & .292 & .044 & .000 \\
\hline Allodynia \% & 31.9 & - & - & 41.8 & - & - & .024 & - \\
\hline Anosmia/ageusia \% & 74.5 & 73.2 & .479 & 19.1 & 23.1 & .073 & .000 & .000 \\
\hline Sore throat, rhinorrhea \% & 62.2 & 70.4 & .152 & 41.8 & 54.4 & .000 & .000 & .006 \\
\hline $\begin{array}{l}\text { Stinging, burning, tearing in } \\
\text { the eyes }\end{array}$ & 63.2 & 64.6 & .481 & 44.8 & 49.8 & .063 & .000 & .011 \\
\hline Use of analgesics \% & 84.3 & 79.8 & .257 & 82.8 & 69.8 & .000 & .389 & .042 \\
\hline No response to analgesics \% & 16.5 & 22.4 & .227 & 7.1 & 8.0 & .353 & .002 & .002 \\
\hline $\begin{array}{l}\text { Partial response to analgesics } \\
\leq 50 \%) \%\end{array}$ & 38.1 & 38.8 & .530 & 25.5 & 37.6 & .000 & .006 & .484 \\
\hline $\begin{array}{l}\text { Improved with analgesics > } \\
50 \%\end{array}$ & 32.0 & 25.4 & .231 & 26.1 & 27.4 & .362 & .129 & .436 \\
\hline $\begin{array}{l}\text { Completely recovered with } \\
\text { analgesics } \%\end{array}$ & 12.4 & 11.9 & .568 & 39.2 & 26.2 & .000 & .000 & .008 \\
\hline
\end{tabular}

${ }^{a}$ Diagnosed by positive PCR test; ${ }^{\mathrm{b}}$ No COVID-19 symptoms or diagnosis; $+\mathbf{p}(1-3) p$ value between the patients with prior headache with or without positive COVID-19 test; $\neq \mathbf{p}(\mathbf{2}-\mathbf{4}) p$ value between the patients with no prior headache with or without positive COVID-19 test (significant test values are marked as bold)

The great majority of COVID-19 patients with previous headaches $(79.5 \%, 89$ out of 112 responders) reported that their new emerging headaches during the infection period were different from their usual headaches; among them, 50\% (56 participants) disclosed that this new headache was totally different, whereas $29.5 \%$ (33 participants) reported some differences despite some similar properties resembling previous headache features. On the other hand, among the participants without COVID-19 diagnosis but with previous headaches, $62.7 \%$ (785 out of 1252 responders) disclosed that their headaches were identical to the pre-existing episodes. Among them, only $13.9 \%$ (174 participants) reported entirely different attacks, whereas $23.4 \%$ (293 participants) reported partly different headaches.

Severity of the previous headaches did not relate to receiving COVID-19 diagnosis; COVID-19 positive patients had reported headaches of mild intensity in $26.6 \%$ (34 out of 128 responders), moderate-intensity in $47.7 \%$ (61 participants), severe in $23.4 \%$ and very severe (dependent to bed/hospital) in 2.3\% (3 participants). The corresponding rates of headache intensity were $27.7 \%$ (484 out of 1747 responders), 46.7\% (816 participants), 21\% (367 participants), and 4.6\% (80 participants) in COVID-19 negative participants.

Among all 262 patients diagnosed with COVID-19, $40 \%$ (104 participants) reported high fever (over $38^{\circ} \mathrm{C}$ ), 49.2\% (128 participants) prominent cough, 48.3\% (125 participants) sore throat and $33.5 \%$ (87 participants) shortness of breath. On the other hand, diarrhea/stomachache was present in 57.7\% (150 participants) and lastly anosmia/ageusia was present in 60.4\% (157 participants) of this group. There were 59 (22.5\%) COVID-19 positive patients who had previous headaches, who did 
not experience headaches during the pandemic period of 2 months at the time of their participation in the survey. At least one close contact with the disease was present in $65.4 \%$ (170 participants) of the COVID-19 positive patients and $90.1 \%$ of the patients lived under quarantine.

\section{Triggers of headache in the study population}

The triggers of headache reported by the participants are shown comparatively in COVID-19 positive and negative cases in the Fig. 2; the second part of this figure shows the triggers reported by healthcare workers compared to the others. We noticed that stress was the most common trigger up to $30 \%$ of the participants as seen as in the Fig. 2 and social isolation triggered headache noteworthy in patients without COVID-19. On the other hand, the patients diagnosed with COVID-19 reported also infection itself and the drugs as triggers of headache.

\section{The course of headache in participants during the pandemic}

The reported changes in the headache course after the pandemic were shown in Fig. 3. Among the responding 1886 patients, $23.3 \%$ reported an increase in severity of headache, $28.7 \%$ reported an increase of the headache duration, and lastly $14 \%$ reported deterioration in the accompanying symptoms. The decreased headache frequency in $12.3 \%$ of the group was remarkable.

Our analysis showed that among the patients with preexisting migraine diagnosis, the frequency of pulsating headache character decreased from $60.5 \%$ (328 out of $542 \mathrm{pts}$ ) to $55 \%$ (22 out of $40 \mathrm{pts}$ ) in those receiving COVID-19 diagnosis. However, this latter rate was still significantly higher in comparison to $34.1 \%$ (31 out of 91 pts) compared to COVID-19 positive patients without previous headache $(p=0.033)$.

The worsening of previous headache and analgesic unresponsiveness did not show any difference in relation to ages of the participants with COVID-19.

\section{Differentiating variables of COVID-19 related headache}

The important differentiating variables for COVID-19 infection patients suffering from headaches were summarized in Table 2 and graphed as Fig. 4, with calculated Odds ratios (OR). According to our findings, the presence of bilateral headache, duration over $72 \mathrm{~h}$, male gender, analgesic resistance, gastrointestinal symptoms and anosmia/ageusia showed increased risk of having headache related to COVID-19 infection.

Additionally, binary logistic regression was computed with significant headache variables which could be observed in everyday practice to distinguish COVID-19 related headaches. The model summary showed a $-2 \log$ likelihood ratio of 1003.6 (Nagelkerke R Square: 0.105) and using this model $88.6 \%$ of the COVID-19 patients could be classified correctly with a cut off value of 0.5 . We did not enter infection-related features in this analysis, because they are clear clues for COVID-19 and do not appear in control cases. Our findings disclosed that bilateral headache, duration over $72 \mathrm{~h}$, male gender, and analgesic resistance were important variables to differentiate between COVID-19 positive patients from negative ones, showing statistical levels as $p=0.04$ for long duration of headaches and $p<0.000$ for the remaining variables (Supplementary Table 1).

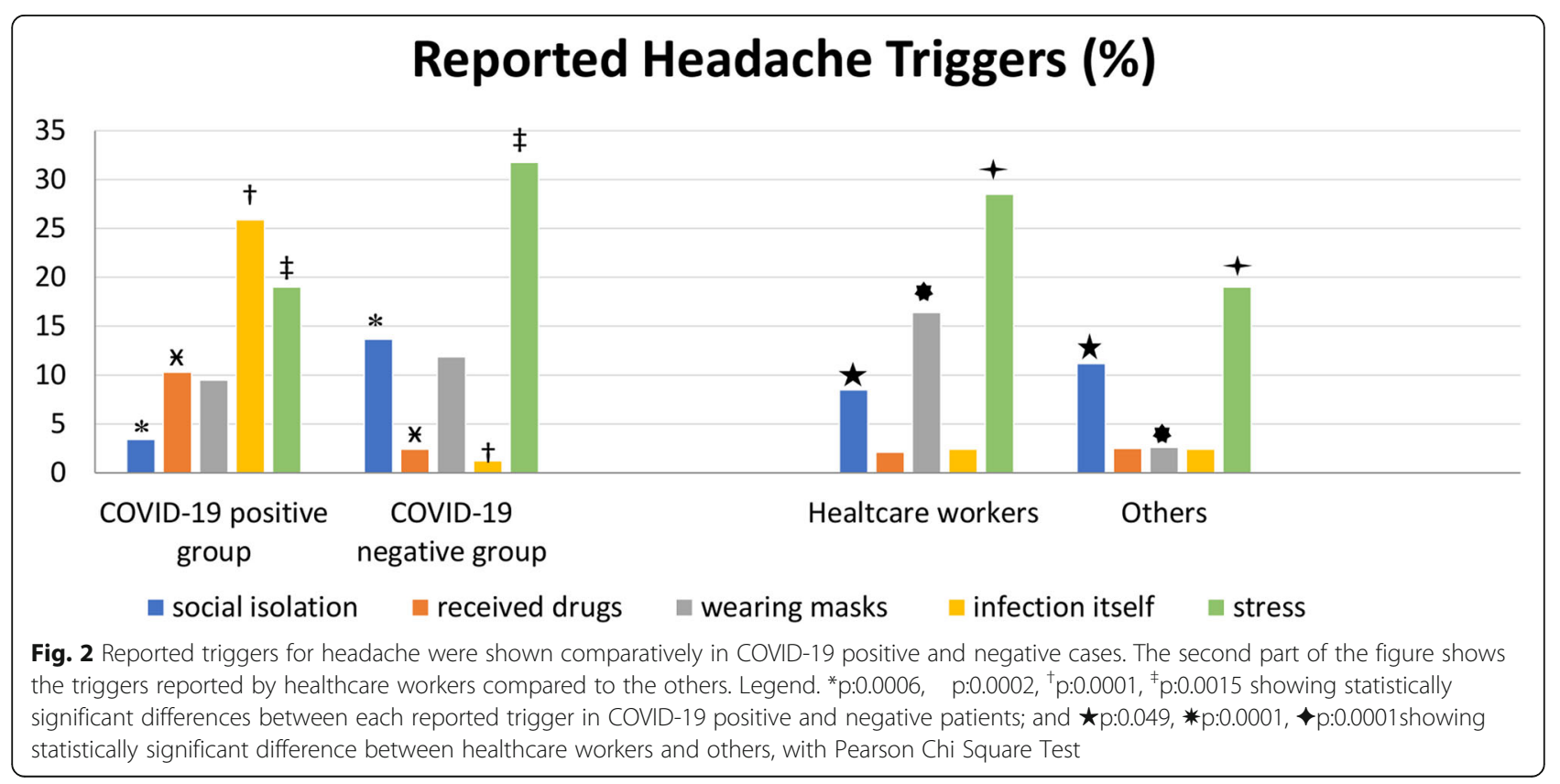




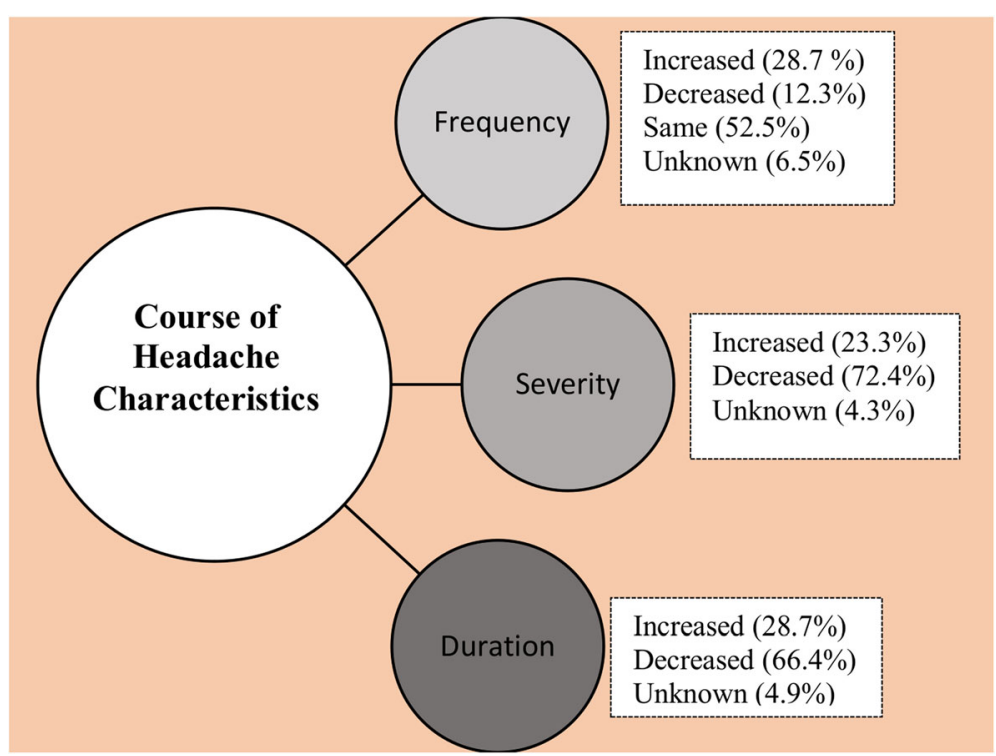

Fig. 3 The course of headache characteristics

\section{Discussion}

This first careful analysis of emerging headache characteristics in the pandemic showed that COVID-19 related headaches are more closely associated with anosmia/ ageusia and gastrointestinal complaints, in comparison to other usual infection findings. Moreover, bilateral headache, duration over $72 \mathrm{~h}$, male gender, and analgesic resistance are highly important variables to differentiate between COVID-19 positive patients from negative ones. Although we expected prominent worsening of primary headaches due to the pandemic-related problems, this happened only for less than $1 / 3$ of participants, mostly related to stress.

\section{Gender difference of COVID-19 related headaches}

Despite the well-known predominance of headaches in females and the fact that more than $2 / 3$ of participants answering our survey consisted of females, COVID-19

Table 2 Odds ratios of differentiating variables of the headache in patients with COVID-19 diagnosis

\begin{tabular}{llll}
\hline Headache characteristics & $\begin{array}{l}\text { COVID-19 positive } \\
\text { n (\%) }\end{array}$ & $\begin{array}{l}\text { No COVID-19 } \\
\text { n (\%) }\end{array}$ & OR \\
\hline Presence of anosmia/ageusia & $142(74 \%)$ & $282(\% 20)$ & 11.4 \\
Bilateral headache & $166(85 \%)$ & $994(64 \%)$ & 3.37 \\
Analgesic-resistant & $32(\% 18)$ & $116(8 \%)$ & 2.61 \\
Gastrointestinal symptoms & $137(70 \%)$ & $808(53 \%)$ & 2.13 \\
Male gender & $82(\% 40)$ & $437(25 \%)$ & 2.06 \\
Headache duration $>72 \mathrm{~h}$ & $27(13.3 \%)$ & $130(7 \%)$ & 1.93 \\
Headache duration $>48 \mathrm{~h}$ & $55(27 \%)$ & $328(18.5 \%)$ & 1.63 \\
\hline
\end{tabular}

related headaches were reported by male patients at a high rate. This interesting finding is somewhat in line with the predominance of COVID-19 in male gender, with changing reported rates around 56-73\% [12-14]. It could be hypothesized that this reversed gender dominance may relate to comorbidities like atherosclerosis and hypertension which are more frequent in males. However, females with nearly three times higher rates of migraine could still have outnumbered in COVID-19 related headaches $[12,15]$. Therefore, this finding is a striking point that needs further careful elaboration. Given the higher risk of male gender in COVID-19 cases, a protective role of female hormones or of the location of Angiotensin-converting enzyme 2 (ACE-2) on the $\mathrm{X}$ chromosome could be speculated $[9,12]$. Moreover, ACE-2 expression level which is critical for the SARS- CoV-2 entry to the cells was found different between genders [14]. There is some evidence that immune activity is more efficient in females in other viral infections [16]. It is tempting to speculate that SARS$\mathrm{CoV}-2$ may trigger some silenced genes related to innate immunity in the $\mathrm{X}$ chromosome, so two $\mathrm{X}$ chromosomes may serve for a more effective and balanced war against COVID-19 related hyperactivation of immune pathways. Moreover, estrogens and progesterone have antiinflammatory actions partially through inflammasome activation in some models [17]. ACE also affects the display of major histocompatibility complex (MHC) class I and MHC class II peptides [18]. Thus, further work with these gender-related differences may give us some clues to find out novel protective ways against COVID-19. 


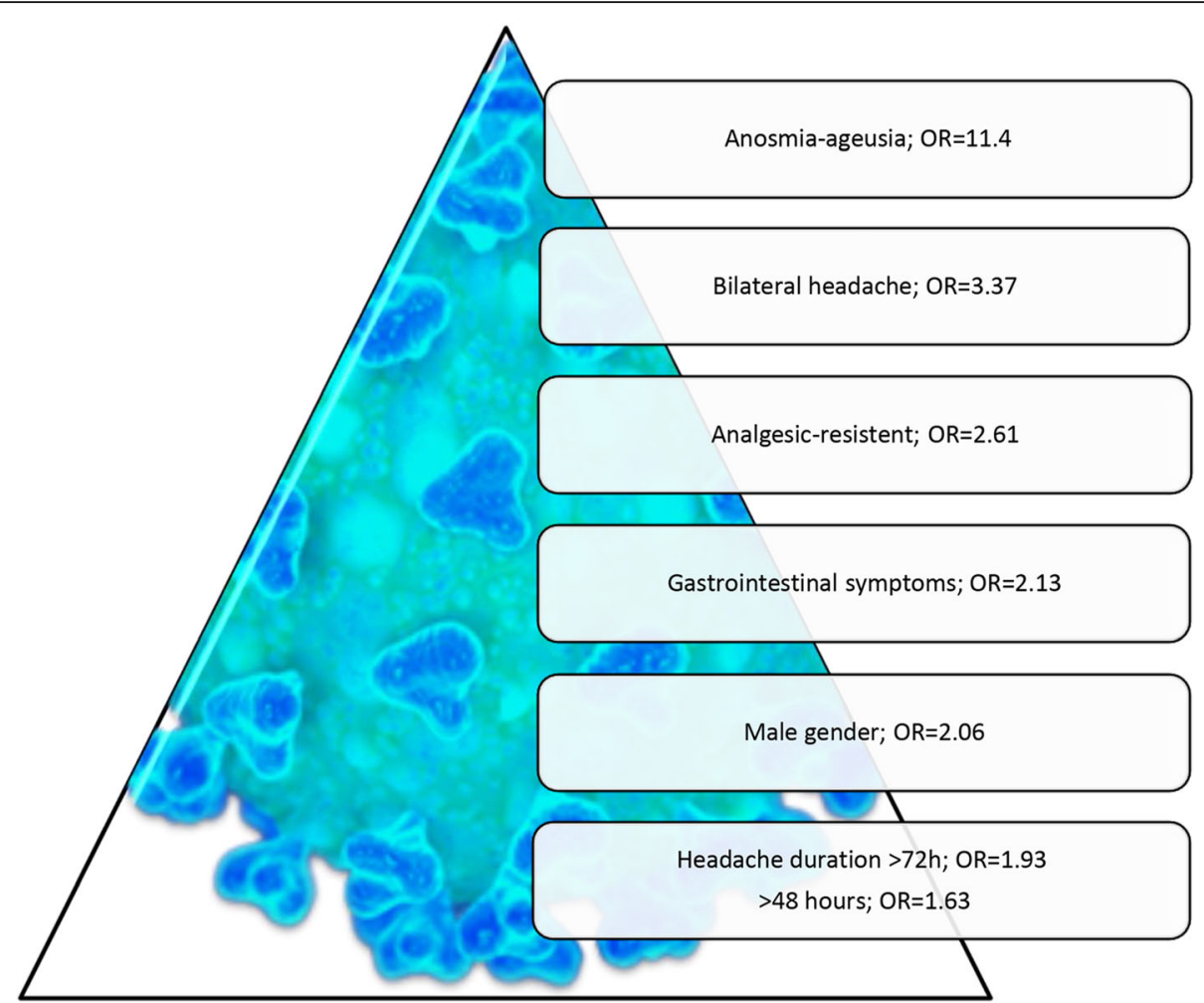

Fig. 4 Odds ratios of differentiating variables related to the presence of headache in patients with COVID-19 diagnosis reporting headache during the pandemic

\section{Possible mechanisms underlying COVID-19 related headaches in the light of our findings}

The underlying mechanisms of headache related to COVID-19 are not clear at this early moment [9]. A direct invasion of trigeminal nerve endings in the nasal or oral cavity by the virus seems one of the most reasonable mechanisms underlying headache according to our results showing the close relation between headache and anosmia/ageusia. Some coronaviruses were shown to be neurotropic and former SARS-CoV has been observed in the human brain $[19,20]$. Therefore, it is highly likely that SARS-CoV-2 may also enter the nervous system via the cranial nerves. Since the first observations in China, there are many worldwide reports showing heterogeneous prevalence figures around $5-85 \%$ of loss of smell $[21,22]$. These profound differences may relate to the viral load differences and a different individual immune response between younger milder symptomatic outpatients, who are eager to report their symptoms, and contrarily severe COVID-19 inpatients with prominent respiratory problems who probably under-report this relatively milder problem. It is well-known that methodological differences exist between questionnaire studies and objective measurements, the former being mostly with lower prevalence. Despite this fact, the rate of anosmia/ageusia was high in our study. These two distinct problems caused by different nerves were not easy to differentiate from each other in daily life, therefore anosmia and ageusia were evaluated together in our study. It was remarkable that nasal obstruction and rhinorrhea were frequently reported but not strictly correlated with anosmia and ageusia according to our results. Although the trans-synaptic transfer of SARS-COV-2 is not proven yet, this possibility of the trans-synaptic route was documented for other coronaviruses [23]. Entrance from the nasal cavity to the olfactory bulb, then spreading to the brainstem via the piriform cortex with both passive diffusion and axonal transport has been demonstrated [24].

Besides the described headache characteristics and COVID-19 related respiratory tract symptoms, abdominal pain and diarrhea should be taken into account to evaluate these patients [3-5, 25]. Our study found a high rate of gastrointestinal symptoms like diarrhea/stomachache in more than half of the COVID-19 cases and also high rates of nausea (71\%) as an accompanying symptom of headache. A previous interesting report from China on non-classical symptoms indicated that $21.6 \%$ of patients had gastrointestinal symptoms, associated with headache, which is a higher number in comparison to patients without gastrointestinal symptoms [26]. The intriguing relationship of headache with gastrointestinal 
symptoms also brings to mind several interesting mechanisms including increased circulating Calcium gene related peptide (CGRP) levels and "gut-brain axis" concept where several inflammatory mediators like Interleukin$1 \beta$ (IL-1 $\beta$ ), Interleukin-6 (IL-6), Interleukin-8 (IL-8), and Tumor necrosis factor- $\alpha$ (TNF- $\alpha$ ), besides gut microbiota, and neuropeptides including CGRP are thought to play a role in this interaction. Another relevant consideration is systemic CGRP increase, possibly induced by both angiotensin II and IL-6 levels, as CGRP is clearly associated with trigemino vascular activation resulting in headache, increased gastrointestinal (GI) motility leading to diarrhea, further triggering inflammation and vascular edema $[9,27]$. Taken together with the unusually high rate of ageusia-anosmia (60.4\%), this data may lead us to the footsteps of the viral pathway in the brain. Thus, we may also suggest that neuronal invasion of this new coronavirus may cause the dysfunction of the network at brainstem sites, in addition to headache. Nausea and vomiting are associated GI symptoms of migraine headache, yet the diarrhea is a distinct GI feature associated with headache in COVID-19, which clearly shows that opposite influencers play role in SARS-CoV2 infected gut and trigeminal nerve.

Headache in relation with a systemic viral infection, (without signs of meningo-encephalitis) is described in the International Classification of Headache Disorders-3. The underlying mechanisms of this entity are not illuminated so far. Regarding its characteristics, diffuse pain of moderate/severe intensity, commonly with fever was noted [11]. However, in our analyses, the association of headache with fever seems not to be decisive (in all COVID-19 patients with headache, only 40\% reported high fever). Also, rhinosinusitis and other respiratory tract symptoms did not seem to explain the headaches in many of these cases, as seen in Table 1 . Therefore, for this emerging COVID-19 related headache, the simplistic view of a "causal" relationship with fever or upper respiratory symptoms is not explanatory.

\section{The course of headaches during the pandemic and reported triggers for headache}

Most of the patients with pre-existing headaches easily noticed that this was a different problem if they had COVID-19 related headache according to our survey. In a recent case report, the authors highlighted the need to consider secondary headaches, related to central nervous system infections in the setting of COVID-19 in patients experiencing refractory headache, even if the patient had chronic migraine [28]. On the other hand, it was also intriguing that $22.5 \%$ of the COVID-19 positive cases with previous headaches did not suffer from headache during the pandemic and during the infection. There is no clear explanation for the lack of headache in these cases; causes related to viral load, transmission route, or individual differences may play a role. Other interesting data were the stabile course (53\%) or even decrease of the attack frequencies $(12 \%)$ and reduced severity of the preexisting headaches in the pandemic period despite the apparent stressful conditions. Social isolation may have helped to avoid stressful social interactions; a healthy diet, and mild sports activities are possible with spending more time at home, also reducing the stress of dailywork life during the pandemic; all these points were possible reasons for the better headache outcome than expected.

The triggers for headaches showed significant differences between participants with and without COVID-19 infection. Headache was triggered more frequently by stress and social isolation in patients without COVID-19 whereas patients diagnosed with COVID-19 reported also infection itself and the drugs as triggers of headache. Furthermore, wearing masks as well as stress both were the main triggers of headache and were more frequently reported by healthcare workers than by the others. Standard hygiene precautions seemed to reduce the risk for the healthcare workers who did not show an increased risk for COVID-19 compared to other participants in our study. It was reported from Germany that an overall seroprevalence of SARS-CoV-2 in a tertiary hospital was low, given the standard hygiene measures taken [29].

\section{Other differentiating features and associations of COVID- 19 related headache}

The clinical features of COVID-19 infection in nonhospitalized adults were reported to be different from hospitalized patients warranting greater awareness of this wider spectrum of clinical symptoms [30]. Headache as the leader of the COVID-19 related neurological symptoms is the most frequent complaint in outpatient clinics [31]. Thus, it is essential to recognize those patients with COVID-19 at the beginning of the visit or even in telemedicine visits. In this study, we provided evidence that long-lasting bilateral headaches over 48 $72 \mathrm{~h}$ and headaches resistant to analgesics suggested the likelihood of being infected by COVID-19 similar to other secondary headaches. The interesting distributions of pulsating and pressing characters in COVID-19 patients, as seen in Table 1 , showed that pulsating type was more pronounced in patients with previous headaches; this may indicate that individual backgrounds are important in the final phenotypic presentation of COVID-related headache. COVID-19 infection may play a synergistic role in nociception using similar pathways of the trigeminovascular complex as the underlying primary headache such as migraine. However, different characteristics like pulsating, pressing, and even stabbing 
quality may indicate that more than one mechanism is involved in COVID-19 related headache emergence. A case report by a headache expert diagnosed with COVID-19 also indicated that several types of headaches can be seen during COVID-19 infection based on a single case (himself) [32]. Our data had a cross-sectional design; and many participants have chosen only one type despite the availability of multiple choices. Another intriguing finding was that photophobia was more frequently experienced by infected participants than those without COVID-19, reaching statistical significance between the subgroups without prior headache. Moreover, osmophobia was more frequently seen in the group with COVID19, which may also be related to the olfactory dysfunction. Further basic studies are needed to clarify the mechanism of COVID-19-related headache and to unravel the mysteries of the environmental factors including viruses for the headache mechanisms, to make the pandemic disaster an opportunity.

\section{Limitations and strengths of the study}

There are some limitations to this study. First of all, we investigated headache characteristics via questionnaire; the results were based on the answers of the patients, with the potential of a reporting bias well known in all survey studies. Secondly, the patients with COVID-19 were not examined by a physician or headache specialist. Furthermore, our questionnaire was a web-based survey, therefore, only individuals who were able to use new technological devices, thus probably younger and educated people could participate in the study. Among the participants, there may also be some patients who were not tested for COVID-19 due to the lack of other accompanying symptoms. Moreover, patients with severe COVID-19, at the time of the survey, could not be included.

The main strength of our study was the participation of a large number of people in a very short time in the increasing phase of the pandemic. We used a detailed dedicated questionnaire investigating various characteristics of previous and current headaches, including also cross-questions to avoid misunderstandings. The answers given by participants were also examined meticulously to minimize discrepancies. Furthermore, the presence of healthcare workers, reaching nearly half of the participants has increased the reliability of the study.

\section{Conclusion}

The COVID-19 pandemic seems to have a particular effect on the characteristics and the course of headaches in individuals with and without COVID-19 diagnosis, according to our findings. We disclosed that having male gender, bilateral, long-lasting headaches, and resistance to analgesics were more frequently seen in people with
COVID-19 infection in conjunction with anosmia/ageusia and gastrointestinal complaints, besides other infection findings. We propose that these features may be diagnostic for COVID-19 infection in the clinical evaluation of headache patients during the pandemic. We think that COVID-19 related headache should be considered as a separate entity, among the infection-related secondary headaches, with this different profile.

\section{Supplementary information}

Supplementary information accompanies this paper at https://doi.org/10. 1186/s10194-020-01188-1.

Additional file 1: Supplementary Table. Logistic regression analysis model to differentiate patients with COVID-19 from those without COVID-19 based on headache characteristics.

\section{Abbreviations}

COVID-19: Coronavirus disease 2019; SARS-CoV 2: Severe acute respiratory syndrome coronavirus 2; ACE-2: Angiotensin converting enzyme; TNFa: Tumor necrosis factor-a; IL-1 $\beta$ : Interleukin-1 $\beta$; IL-6: Interleukin-6; IL8: Interleukin-8; CGRP: Calcium gene related peptide; $\Pi \mathrm{TH}$ : Tension type headache; ICD-3: International Classification of Headache Disorders, 3rd Edition; PCR: Polymerase chain reaction; MHC: Major histocompatibility complex; RR: Relative risk ratio; OR: Odds ratio; Gl: Gastrointestinal

\section{Acknowledgments}

This study was funded by Scientific Research Projects Coordination Unit of Istanbul University with a project number of GAP-36802. The web and handy design of the questionnaire was delicately organized by Vedat Kamer, Sevinc Gülseçen and Başak Kurtuldu from Istanbul University Department of Informatics, to whom the authors are grateful. The authors also thank to the members of the Turkish Neurology Society Headache Group and Global Migraine and Pain Society for supporting the dissemination of the questionnaire.

\section{Authors' contributions}

$H B, A O, E K O, E E, B B$ designed the survey. ME did statistical analyses. All authors drafted the manuscript. The author(s) read and approved the final manuscript.

\section{Funding}

We received support from the Istanbul University Scientific Research Fund at the stages of creating web questionnaires and reaching patients (project number: GAP-36802).

\section{Availability of data and materials}

This study is a web-based questionnaire, the data is not applicable.

Ethics approval and consent to participate

Istanbul University Istanbul Faculty of Medicine Ethics Committee approved the study (17.04.2020/520)

Consent for publication

Not applicable.

\section{Competing interests}

We declare no competing interests.

\section{Author details}

${ }^{1}$ Department of Neurology, Istanbul Faculty of Medicine, Istanbul University, Çapa, Istanbul, Turkey. Department of Neurology and Algology, Gazi University Faculty of Medicine, Besevler, 06510 Ankara, Turkey. ${ }^{3}$ Department of Neurology, Mersin University School of Medicine, Mersin, Turkey. ${ }^{4}$ Department of Infectious Diseases and Clinical Microbiology, Istanbul Faculty of Medicine, Istanbul University, Çapa, Istanbul, Turkey. 
Received: 16 August 2020 Accepted: 25 September 2020

Published online: 13 October 2020

\section{References}

1. Guan WJ, Ni ZY, Hu Y et al (2020) Clinical characteristics of coronavirus disease 2019 in China. N Engl J Med 382:1708-1720

2. Mao L, Jin H, Wang M et al (2020) Neurologic manifestations of hospitalized patients with coronavirus disease 2019 in Wuhan, China. JAMA Neurol 77:1-9

3. Borges do Nascimento IJ, Cacic N, Abdulazeem HM et al (2020) Novel Coronavirus Infection (COVID-19) in Humans: A Scoping Review and MetaAnalysis. J Clin Med 9(4):941

4. Chen T, Wu D, Chen $\mathrm{H}$ et al (2020) Clinical characteristics of 113 deceased patients with coronavirus disease 2019: retrospective study. BMJ 368:m1091

5. Zhang X, Cai H, Hu J et al (2020) Epidemiological, clinical characteristics of cases of SARS-CoV-2 infection with abnormal imaging findings. Int J Infect Dis $94: 81-87$

6. Kaur N, Gupta I, Singh H et al (2020) Epidemiological and clinical characteristics of 6635 COVID-19 patients: a pooled analysis. SN Compr Clin Med 9:1-5

7. Ozdag Acarli AN, Samanci B, Ekizoglu E et al (2020) Coronavirus disease 2019 (COVID-19) from the point of view of neurologists: observation of neurological findings and symptoms during the combat against a pandemic. Arch Neuropsychiatry 57:154-159

8. Pinzon RT, Wijaya VO, Buana RB et al (2020) Neurologic characteristics in coronavirus disease 2019(COVID-19): a systematic review and meta-analysis. Front Neurol 11:565

9. Bolay H, Gul A, Baykan B (2020) COVID-19 is a real headache! Headache. 60: 1415-1421

10. Toptan T, Aktan C, Basari A, Bolay H (2020) Case series of headache characteristics in COVID-19; headache can be an isolated symptom. Headache 60:1788-1792

11. Headache Classification Committee of the International Headache Society (IHS) (2018) The international classification of headache disorders, 3rd edition. Cephalalgia 38:1-211

12. Cheng ZJ, Shan J (2020) 2019 novel coronavirus: where we are and what we know. Infection 48:155-163

13. Yang X, Yu Y, Xu J et al (2020) Clinical course and outcomes of critically ill patients with SARS-CoV-2 pneumonia in Wuhan, China: a single-centered, retrospective, observational study. Lancet Respir Med 8:475-481

14. Zhao Y, Zhao Z, Wang Y, Zhou Y, Ma Y, Zuo W (2020) Single-cell RNA expression profiling of ACE2, the putative receptor of Wuhan 2019-nCov. Am J Respir Crit Care Med 202:756-759

15. Wang Y, Chen Y, Qin Q (2020) Unique epidemiological and clinical features of the emerging 2019 novel coronavirus pneumonia (COVID-19) implicate special control measures. J Med Virol 92:568-576

16. Alon T, Doepke M, Olmstead-Rumsey J, Tertilt M (2020) The Impact of COVID-19 on Gender Equality. Covid Economics Vetted Real Time Papers 4: 62-85

17. Slowik A, Beyer C (2015) Inflammasomes are neuroprotective targets for sex steroids. J Steroid Biochem Mol Biol 153:135-143

18. Bernstein KE, Khan Z, Giani JF, Cao DY, Bernstein EA, Shen XZ (2018) Angiotensin-converting enzyme in innate and adaptive immunity. Nat Rev Nephrol 14:325-336

19. Ding $Y$, He L, Zhang Q et al (2004) Organ distribution of severe acute respiratory syndrome (SARS) associated coronavirus (SARS-CoV) in SARS patients: implications for pathogenesis and virus transmission pathways. J Pathol 203:622-630

20. Gu J, Gong E, Zhang B et al (2005) Multiple organ infection and the pathogenesis of SARS. J Exp Med 202:415-424

21. Izquierdo-Dominguez A, Rojas-Lechuga MJ, Mullol J, Alobid I. Olfactory dysfunction in the COVID-19 outbreak. J Investig Allergol Clin Immunol. 2020;30(5). Doi: https://doi.org/10.18176/jiaci.0567. Online ahead of print

22. Baysal-Kirac L, Uysal H (2020) COVID-19 associate neurological complication. Neurol Sci Neurophysiol 37:1-3

23. Mengeling WL, Boothe AD, Ritchie AE (1972) Characteristics of a coronavirus (strain 67N) of pigs. Am J Vet Res 33:297-308

24. Desforges M, Le Coupanec A, Dubeau P, Bourgouin A, Lajoie L, Dube M et al (2020) Human Coronaviruses and Other Respiratory Viruses: Underestimated Opportunistic Pathogens of the Central Nervous System? Viruses 12(1):14. https://doi.org/10.3390/v12010014
25. Huang C, Wang Y, Li XR et al (2020) Clinical features of patients infected with 2019 novel coronavirus in Wuhan, China. Lancet 395:497-506

26. Jin X, Lian JS, Hu JH et al (2020) Epidemiological, clinical and virological characteristics of 74 cases of coronavirus-infected disease 2019 (COVID-19) with gastrointestinal symptoms. Gut. 69:1002-1009

27. Falkenberg K, Bjerg HR, Olesen J (2020) Two-hour CGRP infusion causes gastrointestinal hyperactivity: possible relevance for CGRP antibody treatment. Headache. 60:929-937. https://doi.org/10.1111/head.13795

28. Arca KN, Starling AJ (2020) Treatment-refractory headache in the setting of COVID 19 pneumonia: migraine or Meningoencephalitis? Case report. SN Compr Clin med:1-4. https://doi.org/10.1007/s42399-020-00369-y Online ahead of print

29. Korth J, Wilde B, Dolff S et al (2020) SARS-CoV-2-specific antibody detection in healthcare workers in Germany with direct contact to COVID-19 patients. J Clin Virol 128:104437

30. Sharon $\mathrm{H}$, Bergquist $\mathrm{CP}$, Roberts $\mathrm{DL}$, O'Keefe JB, Tong EJ, Zreloff J, Jarrett $T L$, Moore MA (2020) Non-hospitalized adults with COVID-19 differ noticeably from hospitalized adults in their demographic, clinical, and social characteristics. SN Compr Clin Med:1-9. https://doi.org/10.1007/s42399-02000453-3 Online ahead of print

31. Baykan B, Ertas M, Karli N et al (2007) The burden of headache in neurology outpatient clinics in Turkey. Pain Pract 7:313-323

32. Belvis R (2020) Headaches during COVID-19: my clinical case and review of the literature. Headache, 60:1422-1426

\section{Publisher's Note}

Springer Nature remains neutral with regard to jurisdictional claims in published maps and institutional affiliations.
Ready to submit your research? Choose BMC and benefit from:

- fast, convenient online submission

- thorough peer review by experienced researchers in your field

- rapid publication on acceptance

- support for research data, including large and complex data types

- gold Open Access which fosters wider collaboration and increased citations

- maximum visibility for your research: over $100 \mathrm{M}$ website views per year

At $\mathrm{BMC}$, research is always in progress.

Learn more biomedcentral.com/submissions 